W-Folie des Ofens dienen. Da diese aber stromdurchflossen ist und deshalb ein Potentialgefälle von 4,5 Volt besitzt, wird in den Ofen ein zweiter W-Zylinder von $7 \mathrm{~mm}$. Durchmesser geschoben, der nur mit dem einen Ende des äußeren Zylinders leitend verbunden ist und sich also auf konstantem Potential befindet. Zwischen dem Pt-Draht und diesem inneren Zylinder wird nun die variable, elektronenbeschleunigende Spannung angelegt. Zur Feststellung der Strahlung, die von den durch den Elektronensto $B$ angeregten Atomen ausgesandt wird, djent in der üblichen Weise die Messung des Photostromes, der durch die Strahlung an einer Auffangplatte ausgelöst wird. Diese Platte ist außerhalb des Ofens, aber innerhalb des wassergekuhlten Metallgefäßes, das alle Teile einschließlich Öfen umschließt, so angebracht, daß die aus dem Ofen kommende Strahlung auf sie auftrifft. Vor der Platte befindet sich ein Drahtnetz, das gegen die Platte positiv geladen ist, so daß die Photoelektronen, die diese verlassen, beschleunigt werden. Der Photostrom wird mit einem Elektrometer gemessen. Vor dem Drahtnetz ist in besonderer Weise eine Anordnung getroffen, durch die geladene Teilchen, die etwa vom Ofen her kommen könnten, durch elektrische Felder aufgefangen werden. Die Apparatur ist also lediglich geeignet zum Nachweis von Strahlungseffekten, während Ionisation nicht nachweisbar ist.

Gemessen wird nun in der üblichen Weise der lichtelektrische Strom in Abhängigkeit von der Elektronen beschleunigenden Spannung. Dabei ergeben sich Kurven, die bei bestimmten Spannungen sehr scharf ausgesprochene Knicke zeigen. Diese Spannungen ergaben sich zu $\mathrm{r}_{0} \mathrm{I}_{5}, 12,02, \mathrm{x}_{2}, 70, \mathrm{I}_{3}, 00, \mathrm{I}_{3}, \mathrm{I}_{7}, \mathrm{I}_{3}, 27$ und $\mathrm{x}_{3}, 54$ Volt mit einem Fehler $<0,05$ Volt. Dagegen traten die für $\mathrm{H}_{2}$ charakteristischen, kritischen Spannungen nicht auf. Die Deutung der gefundenen Spannungen vom Standpunkte der Bohrschen Theorie des $\mathrm{H}$-Atoms ist nun leicht. Es sind dies die in Volt umgerechneten Arbeiten, die zu leisten sind, um das Elektron des H-Atoms von der einquantigen Bahn der Reihe nach auf die zweiquantige, dreiquantige usw. Bahn zu heben. Diese Arbeit ist für die n-quantige Bahn:

$$
W_{n} \nabla_{n}=h R\left(\frac{\mathbf{I}}{\mathbf{x}^{2}}-\frac{\mathbf{I}}{n^{2}}\right) \text {, }
$$

wo $h$ das Plancksche Wirkungsquantum und $R$ die Rydbergkonstante bedeutet. Für $n=\infty$ berechnet sich diese Arbeit, d. h. die Ionisierungsarbeit, durch die Gleichung $W_{n}=e \nabla_{n}$ in Volt umgerechnet, zu $V_{\infty}=13,52$ Volt. Wir haben also:

$$
\begin{aligned}
& V_{2}=\mathrm{I} 3,5^{2}\left(\frac{\mathrm{I}}{\mathrm{I}^{2}}-\frac{\mathrm{I}}{\mathrm{2}^{2}}\right)=\frac{3}{4} \cdot \mathrm{I} 3,5^{2}=\mathrm{I} \mathrm{I}, \mathrm{I} 4, \\
& V_{3}=\mathrm{I} 3,52\left(\frac{\mathrm{I}}{\mathrm{I}^{2}}-\frac{\mathrm{I}}{\mathrm{3}^{2}}\right)=\frac{8}{9} \cdot \mathrm{I} 3,5^{2}=\mathrm{I} 2,02, \\
& V_{4}=\mathrm{I} 3,52\left(\frac{\mathrm{I}}{\mathrm{I}^{2}}-\frac{\mathrm{I}}{4^{2}}\right)=\frac{\mathrm{I}}{\mathrm{I} 6} \cdot \mathrm{I} 3,5^{2}=\mathrm{I} 2,69, \\
& V_{5}=\mathrm{I} 3,52\left(\frac{\mathrm{I}}{\mathrm{I}^{2}}-\frac{\mathrm{I}}{5^{2}}\right)=\frac{24}{25} \cdot \mathrm{I} 3,52=\mathrm{I} 3,00, \\
& V_{6}=\mathrm{I3}, 5^{2}\left(\frac{\mathrm{I}}{\mathrm{I}^{2}}-\frac{\mathrm{I}}{6^{2}}\right)=\frac{35}{36} \cdot \mathrm{I} 3,5^{2}=\mathrm{I} 3, \mathrm{I} 7, \\
& V_{7}=\mathrm{I} 3,5^{2}\left(\frac{\mathrm{I}}{\mathrm{I}^{2}}-\frac{\mathrm{I}}{7^{2}}\right)=\frac{4^{8}}{49} \cdot \mathrm{I} 3,5^{2}=\mathrm{I} 3,26 .
\end{aligned}
$$

Man sieht also, daß die gemessenen Werte mit den von der Theorie geforderten innerhalb der kleinen Fehlergrenze vallkommen übereinstimmen. Die Tatsache, daß auch bei der Ionisierungsspannung 13,54 Volt ein Knick beobachtet wird, etscheint dem Verfasser plausibel auf Grund der bekannten Erscheinung, daß oberhalb der Ionisierungsspannung das Wasserstoffspektrum viel stärker herauskommt. Die Wiedergabe der Kurven in der Originalarbeit zeigt deutlich das Vorhandensein der Knicke, die für die niedrigeren Spannungswerte auch sehr scharf ausgeprägt, bei der Werten von I3,o Volt an aber doch nur schwer erkennbar sind. Man wird aber den Verfassern glauben, daß sie sich bei deren Festlegung durch die Kenntnis der Knickstellen nicht haben subjektiv beeinflussen lassen. Das Resultat der Arbeit stellt, wie die Verfasser mit Recht sagen, eine erfreuliche Bestätigung der Bohr schen Theorie des Wasserstoffatoms dar, doch möchten wir meinen, daB der Hauptwert der Arbeit in der Erweiterung der experimentellen Möglichkeiten liegt, indem nun die hier entwickelte Methode auf andere Gase wie $\mathrm{N}_{2}$ und $\mathrm{O}_{2}$ angewandt werden kann, bei denen die zu erwartenden Anregungsspannungen der Atome noch unbekannt sind. Derartige Versuche sind nach Angabe der Verfasser in Angriff genommen.

\section{W. Grotrian}

Das Resultat einiger Versuche über fraktionierte Krystallisation gewöhnlichen Bleis, die nicht weiter fortgesetzt werden konnten, wird von R. H. ATKINSoN (Nature vom 5. April 1924) mitgeteilt: DaB Verf, durch fraktionierte Krystallisation Proben verschiedenerDichte exhalten hatte, hat er in einem Brief an Nature (25. August 1923) berichtet. Er hat jetzt die Dichten beider Proben und die betreffenden Atomgewichte durch Ubberführung des Bleis in Bleisulphat bestimmt.

$\begin{array}{lccc}\text { Dichte: } & \text { II,358 } & - & \text { II,327 } \\ \text { Atomgewicht: } & 207,07 & 207, \text { I } 5 & 207,19\end{array}$
Die Unterschiede sind also noch so klein, daß diese Zahlen einer Prüfung bedürfen, ehe man weitere Schlüsse aus ihnen ziehen kann. Daß die Unterschiede nicht durch Verunreinigung durch Wismut bzw. Thallium entstanden sind, ist durch Identifikation des Schmelzpunktes bewiesen.

Über den Zusammenhang des Potentialgradienten und der Zahl großer Ionen in der Atmosphäre berichtet J. J. Nolan. (Nature vom 5. April 1924.) Er hat auf Grund eigner Beobachtungen festgestellt, daB die Kurven für den Potentialgradienten und die $\mathrm{Zahl}$ großer Ionen sich im allgemeinen weitgehend ähneln. Da eine Vergrößerung der Zahl der großen Ionen die Verklejnerung der Zahl der beweglicheren kleinen und damit eine Vergrößerung des Widerstandes bedingt, muß auch der Potentialanstieg steiler werden, wodurch sich die Ähnlichkeit der Kurven erklären läßt.

v. STMson.

\section{Berichtigungen.}

In dem von BALDUS erstatteten Referat (Heft $2 \mathrm{I}$, S. 4I6, I. Spalte) ist Yungia versehentlich als Borstentier bezeichnet statt als Strudelwurm. Es soll heißen: "Yungia kriecht entweder mit Hilfe seiner Wimpern" und weiter unten:,$\ldots$ sendet Impulse in die Nervenstränge."

In dem Referat über die Thermik der Alpenseen (Heft 22, S. 440) muB es S. $44 \mathrm{I}$, I. Spalte, Zeile I9 von oben heißen: $12 \mathrm{~cm}$ statt $\mathrm{I}_{2} \mathrm{~m}$. 